\title{
Joins of subalgebras and normals in 0-regular varieties
}

\author{
Nicholas McConnell and Tim Stokes
}

Dedicated to Brian Davey on the occasion of his 65th birthday

\begin{abstract}
In any 0-normal variety (0-regular variety in which $\{0\}$ is a subalgebra), every congruence class containing 0 is a subalgebra. These "normal subalgebras" of a fixed algebra constitute a lattice, isomorphic to its congruence lattice. We are interested in those 0 -normal varieties for which the join of two normal subalgebras in the lattice of normal subalgebras of an algebra equals their join in the lattice of subalgebras, as happens with groups and rings. We characterise this property in terms of a Mal'cev condition, and use examples to show it is strictly stronger than being ideal determined but strictly weaker than being 0-coherent (classically ideal determined) and does not imply congruence permutability.
\end{abstract}

\section{Introduction}

1.1. Background on 0 -normal varieties. Let $\mathcal{V}$ be a variety of algebras with nullary operation the distinguished zero element 0 . If for each congruence $\rho$ on each $A \in \mathcal{V}$, the $\rho$-class containing 0 determines $\rho$, then $\mathcal{V}$ is a 0 -regular variety. There is a well-known Mal'cev condition for 0-regularity; see [5]. If also $\sigma(0,0, \ldots, 0)=0$ is an identity for each operation $\sigma$ in the signature of $\mathcal{V}$, then $\mathcal{V}$ is a 0 -normal variety, as in [11]. Evidently 0-normality is equivalent to requiring that the $\theta$-class containing 0 of any congruence $\theta$ on any $A \in \mathcal{V}$ is a subalgebra. Such 0-normal varieties abound, and include any variety of groups or (not necessarily associative) rings, along with many others.

If $\mathcal{V}$ is a 0-regular variety, then for any $A \in \mathcal{V}$, we call the congruence classes containing 0 its normals. In groups the normals are exactly the normal subgroups, while in rings they are the ideals. We write $I \triangleleft A$ if $I$ is a normal in $A \in \mathcal{V}$. (These are called "ideals" in [5], a term which now has a different meaning in general algebra.) For $I \triangleleft A$, let $\rho_{I}$ denote the corresponding congruence on $A$; we write $A / I$ rather than $A / \rho_{I}$, in keeping with the tradition for groups, rings and so on, and write $a I$ for the $\rho_{I}$-class containing $a \in A$.

1.2. Background on EQ-structures. Varieties of EQ-structures are used to prove many of the facts below, so we give some background on these. In [4], the notion of an EQ-monoid is defined, where they were called "E-monoids".

Presented by T. Kowalski.

Received October 23, 2013; accepted in final form June 14, 2014.

2010 Mathematics Subject Classification: Primary: 08BO5; Secondary: 03C05.

Key words and phrases: 0-regular variety, ideal determined variety, Mal'cev condition, EQ-monoid. 
(We adopt the "EQ" prefix as it is consistent with more recent usage as in [12] and elsewhere, and does not conflict with unrelated uses of the term "Esemigroup".) For the examples given here, we only need the results relevant to the commutative case, so we make this assumption throughout.

Thus, for current purposes, an EQ-monoid is a commutative monoid $(A, \cdot, 1)$ in which there is a submonoid $L_{A}$ that is a (meet-)semilattice for which, for every $a, b \in A$, there is a largest element $e \in L_{A}$ such that $e a=e b$. We use the notation $(a \bowtie b)$ for this largest element.

EQ-monoids can be defined purely equationally as the variety of (commutative) monoids with an additional binary operation $\bowtie$, either finitely or in terms of the following axiom scheme: for all $a, b \in A$,

- $(a \bowtie a)=1$, and

- $f(a)(a \bowtie b)=f(b)(a \bowtie b)$ for all derived unary operations $f$ on $A$.

So the class of EQ-monoids is a variety. Indeed, it is 0-normal with respect to the distinguished nullary operation 1 since $\{1\}$ is always a subalgebra, and $d(x, y)=(x \bowtie y)$ is a difference term witnessing 0-regularity; see [4].

In any EQ-monoid $A$, we can define $a \wedge b=(a \bowtie b) a$, which is a (meet-) semilattice operation on $A$. As shown in [4], the normals of an EQ-monoid have a simple description: they are the filters with respect to $\wedge$ that contain 1 ; we call these normal filters. In the case of a finite EQ-monoid, it is easy to see that every normal filter is principal, and indeed is of the form $\langle\alpha\rangle=\{a \in A \mid \alpha \leq a\}$ for some $\alpha \in L_{A}$ : simply let $\alpha$ be the $\wedge$-meet of all elements of the filter (one of which is 1 , and so $\alpha \in L_{A}$ since $a \wedge 1=(a \bowtie 1) \in L_{A}$ for all $\left.a \in A\right)$.

An EQ-semilattice is an EQ-monoid that is multiplicatively a semilattice. An example is the power set of a topological space $X$ with open sets $\mathcal{O}$, equipped with the semilattice operation of intersection and the operation $\bowtie$ in which $S \bowtie T$ is the largest element $U$ of $\mathcal{O}$ for which $S \cap U=T \cap U$ (see Theorem 6 in [4]), which is nothing but the interior of $(S \cap T) \cup(\bar{S} \cap \bar{T})$, where $\bar{S}$ is the complement of $S$ in $X$. Since $S \bowtie 1=S$ for every open set $S$, it follows that in such an example $A$ (or even a subalgebra of such an example), $L_{A}$ consists of the open sets in $A$. Moreover, for $\alpha \in L_{A}$, a principal normal filter $\langle\alpha\rangle$ consists of all subsets of $A$ containing $\alpha$ (since $\alpha \wedge a=\alpha$ if and only if $\alpha \subseteq a$, as is easily seen). Indeed, every EQ-semilattice has a representation in terms of topological spaces: all are embeddable in an example of this kind.

Given the definition of EQ-semilattices, it is easy to see that the variety of Brouwerian (or Heyting) semilattices is term equivalent to the variety of EQ-semilattices satisfying the law $(x \bowtie 1)=x$, under the translations

$$
(x \rightarrow y) \Leftrightarrow(x y \bowtie x), \quad(x \bowtie y) \Leftrightarrow(x \rightarrow y)(y \rightarrow x) .
$$

So EQ-semilattices generalise Brouwerian semilattices.

An EQ-structure is an EQ-monoid $(A, \cdot, 1)$ equipped with one or more additional regular operations $\rho$ of arbitrary arity $n$ for which, for all $a_{i} \in A$ and 
$\alpha \in L_{A}$

$$
\alpha \rho\left(a_{1}, \ldots, a_{n}\right)=\alpha \rho\left(\alpha a_{1}, \ldots, \alpha a_{n}\right) .
$$

It is shown in [4] that the earlier axiom scheme can be extended to define EQ-structures if one includes such additional operations $\rho$ in the definition of the derived unary operation $f$. Moreover, it is shown in [12] that the congruences of $A$ as an EQ-monoid extend to the additional regular operations as well (so the normals in an EQ-structure are precisely those of its underlying EQ-monoid).

Suppose $(A, \cdot, \bowtie, \vee)$ is an EQ-structure with $\vee$ binary, such that the following law holds: $(\alpha \vee \beta) x=\alpha x \vee \beta x$ for all $\alpha, \beta \in L_{A}$ and $x \in A$. Then we say $A$ is a distributive $E Q$-structure. This concept was defined in greater generality in [4], where it was noted that the term $\rho(x, y, z)=(x \bowtie y) z \vee(y \bowtie z) x$ witnesses congruence permutability of the variety of all distributive EQ-structures. (A law involving elements of $L_{A}$ can be viewed as a general law by replacing $\alpha \in L_{A}$ with $(x \bowtie 1)$, for $x \in A$.)

A distributive EQ-lattice is an EQ-semilattice that is also a distributive lattice under its multiplicative (meet-) semilattice order. An example is the EQ-semilattice of subsets of a topological space, equipped also with union. A distributive EQ-lattice $A$ is a regular distributive EQ-structure since trivially $\alpha(a \vee b)=\alpha(\alpha a \vee \alpha b)$, and $(\alpha \vee \beta) a=\alpha a \vee \beta a$ for all $\alpha, \beta \in L_{A}$ and $a \in A$.

1.3. The various joins of normals. The normals of an algebra $A$ in a 0 regular variety $\mathcal{V}$ form a lattice $\mathcal{I}(A)$ in which meet is intersection, isomorphic to the lattice of congruences. Let us denote the join of any two normals $I, J$ of some $A \in \mathcal{V}$ by $I+J$, so $\rho_{I+J}=\rho_{I} \vee \rho_{J}$.

Let $M \leq A$ denote that $M$ is a subalgebra of $A$. The lattice $\mathcal{S}(A)$ of subalgebras of $A$ has meet equal to intersection, and we denote the join of subalgebras $S, T$ by $S \vee T$. In the 0 -normal case, the subalgebra join of two normals is their join in the lattice of normals if and only if $\mathcal{I}(A)$ is a sublattice of $\mathcal{S}(A)$ (which holds in all groups for example).

There is a further construction that provides an intermediate notion of "join". For $A \in \mathcal{A}$, a 0-regular variety, with $S$ a subalgebra of $A$ and $I \triangleleft A$, define $S \cdot I=\bigcup\{s I \mid s \in S\}$. Of course, $S \cup I \subseteq S \cdot I$, and if $S \leq A$, then it is a familiar fact of general algebra that $S \cdot I \leq A$ also. (The subalgebra $S \cdot I$ appears in the 0-regular version of the general algebraic form of the second isomorphism theorem.) For us, the following observation is fundamental.

Proposition 1.1. Suppose $I, J \triangleleft A$ in a 0 -regular variety $\mathcal{A}$. Then $I \cdot J \subseteq I+J$. If also $M \leq A$ and $\mathcal{A}$ is 0-normal, then $M \vee J \subseteq M \cdot J$, so in particular, $I \vee J \subseteq I \cdot J \subseteq I+J$

Proof. If $a \in I \cdot J$, then $a \rho_{J} i$ for some $i \in I$, so $(a, 0) \in \rho_{J} \circ \rho_{I} \subseteq \rho_{J} \vee \rho_{I}=\rho_{I+J}$, that is, $a \in I+J$. In the 0 -normal case, $M \vee J \subseteq M \cdot J$ since $M \cdot J$ is a subalgebra containing $M \cup J$. 
In groups, it happens that if $M$ is a subgroup of $G$ and $I \triangleleft G$, then $M \cdot I=$ $M I$, the product of the subgroups $M$ and $I$, and their join in the lattice of subgroups. In particular then, the lattice of normal subgroups is a sublattice of the lattice of subgroups. However, it turns out that groups and rings are quite special in this regard.

Consider the topological space $X=\{a, b, c\}$ equipped with the collection of open sets $\mathcal{O}=\{\emptyset,\{a, b\},\{c\}, X\}$. Turn $2^{X}$ into an EQ-semilattice under intersection as above. It has as a subalgebra (hence, an EQ-semilattice) $A=$ $\{\emptyset,\{a\},\{a, b\},\{c\}, X\}$ (which is clearly closed under intersections and contains $\mathcal{O}$, and hence is closed under $\bowtie$ as well), which itself has $\mathcal{O}$ as a subalgebra. (As a poset, $A$ is isomorphic to $N_{5}$, the smallest non-modular lattice.) Let $I=\{\{a, b\}, X\}$ and $J=\{\{c\}, X\}$, the principal normal filters generated by $\{a, b\}$ and $\{c\}$, respectively. Then it is straightforward to verify that $I \cdot J=A$ while $J \cdot I=\mathcal{O}$. So in this case, $I \cdot J$ is "as large as possible" and $J \cdot I$ "as small as possible": $I \vee J=J \cdot I \neq I \cdot J=I+J$.

From this example, we can construct the direct product $A \times A$, which has normals $K_{1}=(I, J)$ and $K_{2}=(J, I)$ defined in the obvious manner. Then it is straightforward to verify that $K_{1} \cdot K_{2}=(I \cdot J, J \cdot I)=(A, \mathcal{O}), K_{2} \cdot K_{1}=(\mathcal{O}, A)$, while $K_{1} \vee K_{2} \subseteq(I \vee J, J \vee I)=(\mathcal{O}, \mathcal{O})$ and $K_{1}+K_{2}=(I+J, J+I)=(A, A)$. So there is an algebra in a 0 -normal variety having normals $K_{1}, K_{2}$ for which $K_{1} \cdot K_{2} \neq K_{2} \cdot K_{1}, K_{1} \vee K_{2}$ is properly contained in both, and both are properly contained in $K_{1}+K_{2}$. Visually:

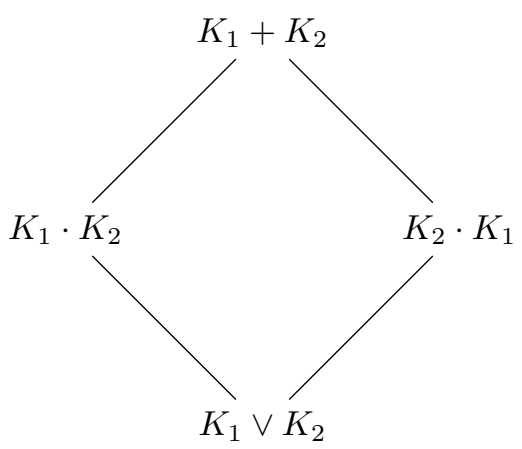

Thus, we have proved the following.

Proposition 1.2. There exists an algebra in a 0 -normal variety having normals $K_{1}, K_{2}$ as pictured, such that all four possible joins are distinct.

1.4. Congruences that permute at zero. Note that if $I \cdot J=I \vee J$ for any two normals $I, J$ of an algebra $A$ in a 0 -normal variety, then $I \cdot J=J \cdot I$ for any two such. This relates to a well-established concept.

Two congruences $\rho, \theta$ on an algebra $A \in \mathcal{A}$, a variety with nullary 0 , are said to permute at zero if $(a, 0) \in \rho \circ \theta$ implies $(a, 0) \in \theta \circ \rho$ and vice versa; equivalently, $(a, 0) \in \rho \vee \theta$ implies the existence of $b \in A$ for which $(b, 0) \in \rho$ and $(a, b) \in \theta$. If any two congruences on $A$ permute at zero, we say $A$ is permutable 
at zero. If every $A \in \mathcal{A}$ is permutable at zero, we say $\mathcal{A}$ is permutable at zero. This notion was first introduced in [6].

In the following, all proofs are either easy or else similar facts have been shown true in [6].

Proposition 1.3. For $A \in \mathcal{V}$, a 0 -regular variety, the following are equivalent.

(1) $I+J=I \cdot J$ for all $I, J \triangleleft A$.

(2) $I \cdot J=J \cdot I$ for all $I, J \triangleleft A$.

(3) $I \cdot J \triangleleft A$ for all $I, J \triangleleft A$.

(4) Any two congruences on A permute at zero.

\section{Join invariance: the definition and a Mal'cev condition}

We say the algebra $A$ in the 0 -normal variety $\mathcal{A}$ is join invariant if for all $I, J \triangleleft A, I+J=I \vee J$, and that a 0 -normal variety is join invariant if every member of it is join invariant. In what follows, we obtain a Mal'cev condition for this property of varieties and relate it to other important possible properties of 0 -normal varieties, such as being ideal determined or congruence permutable.

Proposition 2.1. Let $A \in \mathcal{A}$, a 0 -normal variety. The following are equivalent.

(1) For all $I, J \triangleleft A, I \cdot J=I \vee J$.

(2) $A$ is join invariant.

Proof. If $I \cdot J=I \vee J$ for all $I, J \triangleleft A$, we must have $I \cdot J=I \vee J=J \vee I=J \cdot I$, and so by Proposition $1.3, I \cdot J=I+J$, and congruences permute at zero by that result. Conversely, it is obvious from Proposition 1.1 that if $I+J=I \vee J$ for all $I, J \triangleleft A$, then $I \cdot J=I \vee J$ also.

Mal'cev conditions are important when characterising properties of varieties of algebras. It turns out that join invariance admits such a characterisation.

Theorem 2.2. A 0-normal variety has the join invariance property if and only if there is a term $\delta\left(x_{1}, x_{2}, \ldots, x_{n}, y_{1}, y_{2}, \ldots, y_{m}\right)$ and terms $p_{i}(x, y)$ for $i=1,2, \ldots, n$ and $q_{j}(x, y)$ for $j=1,2 \ldots, m$ such that for all such $i, j$,

$$
\begin{gathered}
p_{i}(x, x)=0, q_{j}(x, 0)=0, \text { and } \\
x=\delta\left(p_{1}(x, y), p_{2}(x, y), \ldots, p_{n}(x, y), q_{1}(x, y), q_{2}(x, y), \ldots, q_{m}(x, y)\right) .
\end{gathered}
$$

Proof. Let $\mathcal{A}$ be a 0 -normal variety with the join invariance property. Then in the free algebra $\mathcal{F}$ in $\mathcal{A}$ on two generators $x, y$, if $\rho_{f, g}$ denotes the least congruence relating $f, g \in \mathcal{F}$ and $I_{f, g}$ the associated normal, we have $x \rho_{x, y} y \rho_{y, 0} 0$, so $x \in I_{x, y} \cdot I_{y, 0}=I_{x, y} \vee I_{y, 0}$. So there is a term $\delta\left(x_{1}, x_{2}, \ldots, x_{n}, y_{1}, y_{2}, \ldots, y_{m}\right)$ such that

$$
x=\delta\left(p_{1}(x, y), p_{2}(x, y), \ldots, p_{n}(x, y), q_{1}(x, y), q_{2}(x, y), \ldots, q_{m}(x, y)\right),
$$


where the $p_{i}(x, y) \in I_{x, y}$ and the $q_{j}(x, y) \in I_{y, 0}$. But for any $f(x, y) \in I_{x, y}$, $0 \rho_{x, y} f(x, y) \rho_{x, y} f(x, x)$, so by [3, Lemma 12.1] (for example), $f(x, x)=0$ in $\mathcal{F}$. Similarly, if $f(x, y) \in I_{y, 0}$, then $f(x, 0)=0$ in $\mathcal{F}$.

Conversely, suppose there are terms as described. Let $A \in \mathcal{A}$ with $I, J \triangleleft A$. Let $a \in J \cdot I$. Then $a \rho_{I} b \in J$, so $p_{i}(a, b) \rho_{I} p_{i}(a, a)=0$ and so $p_{i}(a, b) \in I$ for all $i$. Similarly, $q_{j}(a, b) \rho_{J} q_{j}(a, 0)=0$, so $q_{j}(a, b) \in J$ for all $j$. Hence,

$$
a=\delta\left(p_{1}(a, b), p_{2}(a, b), \ldots, p_{n}(a, b), q_{1}(a, b), q_{2}(a, b), \ldots, q_{m}(a, b)\right) \in I \vee J .
$$

So, $J \cdot I \subseteq I \vee J$, and so $J \cdot I=I \vee J$ by Proposition 1.1. Hence, the variety is join invariant by Proposition 2.1 .

The Mal'cev condition in Theorem 2.2 alone implies the existence of a "subtraction term" $s(x, y)$ satisfying $s(x, x)=0$ and $s(x, 0)=x$ : let

$$
s(x, y)=\delta\left(p_{1}(x, y), p_{2}(x, y), \ldots, p_{n}(x, y), 0,0, \ldots, 0\right) .
$$

This provides an alternative proof that a join invariant 0-normal variety is ideal determined. But it also shows that arbitrary (not necessarily 0-regular) pointed varieties satisfying this Mal'cev condition are subtractive in the sense of [14], or equivalently by [14, Proposition 1.2], all congruences permute at 0 .

\section{Join invariance is stronger than ideal determined}

Ideal determined varieties are defined to be 0-regular varieties in which the normals of algebras are precisely their ideals, which are defined in [6] in terms of closure under certain "ideal terms". Those 0-regular varieties $\mathcal{V}$ in which all congruences on every $A \in \mathcal{V}$ permute at zero are precisely the ideal determined varieties, by a result in [6]; that is, a 0-regular variety is ideal determined if and only if it is subtractive. Any variety of multioperator groups, such as groups or (possibly non-associative) rings, is an ideal determined (0-normal) variety. Other examples include loops and hoops, as well as implication algebras (see [6]), along with others discussed in [11] for example. Ideal determined varieties may be characterised within 0-normal varieties (indeed within 0-regular varieties) in terms of a Mal'cev condition, namely the existence of a term $s(x, y)$ for which $s(x, x)=0$ and $s(x, 0)=x$. (In 0 -normal varieties, this generalises the usual Mal'cev condition for congruence permutability.)

Corollary 3.1. If the 0-normal variety $\mathcal{A}$ has join invariance, then $\mathcal{A}$ is ideal determined.

The converse fails. Consider the variety of implication algebras, defined by the following laws:

(1) $(x \rightarrow y) \rightarrow x=x$;

(2) $(x \rightarrow y) \rightarrow y=(y \rightarrow x) \rightarrow x$;

(3) $x \rightarrow(y \rightarrow z)=y \rightarrow(x \rightarrow z)$. 
Because $(x \rightarrow x)=(y \rightarrow y)$ is a derived identity, $1=(x \rightarrow x)$ is an implicit nullary operation. Implication algebras constitute an ideal determined variety with distinguished nullary operation 1 as is noted in [6]; moreover, it is a 0 -normal variety since $1=1 \rightarrow 1$, so $\{1\}$ is always a subalgebra. All implication algebras can be embedded in the implication algebra $2^{X}$ of all subsets of some set $X$, in which $S \rightarrow T=\bar{S} \cup T$. The variety of implication algebras is given in [6] as an example of an ideal determined variety that is not Mal'cev.

Proposition 3.2. The ideal determined 0-normal variety of implication algebras does not have join invariance.

Proof. Consider the implication algebra $S=2^{X}$ in which $X=\{a, b\}$. For convenience, write $0=\emptyset$, " $a$ " rather than " $\{a\}$ ", " $b$ " rather than " $\{b\} "$, and put $1=X$ (and recall that 1 is the nullary "zero" here). Then the Cayley table for $2^{X}$ as an implication algebra is as follows:

\begin{tabular}{c|cccc}
$\rightarrow$ & 0 & $a$ & $b$ & 1 \\
\hline 0 & 1 & 1 & 1 & 1 \\
$a$ & $b$ & 1 & $b$ & 1 \\
$b$ & $a$ & $a$ & 1 & 1 \\
1 & 0 & $a$ & $b$ & 1
\end{tabular}

It is easy to check that the equivalence relations $\theta_{1}, \theta_{2}$, which partition $S$ into $\{a, 1\},\{0, b\}$ and $\{b, 1\},\{0, a\}$ respectively, are congruences; hence, $I_{1}=\{a, 1\}$ and $I_{2}=\{b, 1\}$ are ideals of $S$. It is also easy to check that $I_{1} \vee I_{2}=I_{1} \cup I_{2}$. But if $J$ is an ideal containing $I_{1} \cup I_{2}$, then because $1 \rightarrow x=x$ by the first law above, we have that $0=1 \rightarrow 0 \rho_{J} a \rightarrow 0=b \in J$, so $0 \in J$ also, and so $J=S$. Hence, $I_{1}+I_{2}=S \neq I_{1} \vee I_{2}$.

\section{0-coherence is stronger than join invariance}

Groups, rings and so on are join invariant varieties, but a rather stronger condition holds in them.

Proposition 4.1. Let $\mathcal{A}$ be a 0 -normal variety, with $A \in \mathcal{A}$. The following are equivalent.

(1) If $I \triangleleft A$ and $M \leq A$, then $M \cdot I=M \vee I$.

(2) If $I \triangleleft A$ and $M \leq A$ with $I \subseteq M$, then $M \cdot I \subseteq M$.

Proof. $(1) \Rightarrow(2)$ : This is obvious.

$(2) \Rightarrow(1)$ : Note that $M \vee I \leq A$, with $I \triangleleft M \vee I$. Then $(M \vee I) \cdot I \subseteq M \vee I$ by assumption, and so $M \cdot I \subseteq(M \vee I) \cdot I \subseteq M \vee I \subseteq M \cdot I$.

The second property above has been considered previously, where varieties globally satisfying it have been called 0 -coherent varieties as in [1], and indeed 
the notion makes sense even in 0-regular varieties (where $\{0\}$, hence any normal, need not be a subalgebra). As a result, we call any algebra in a 0-regular variety 0 -coherent if it satisfies the second condition in the above result.

In [13], 0-coherent varieties are called BIT speciale, (also called classically ideal determined in [14], where they are shown to be ideal determined), and a characterising Mal'cev condition is given for them. In [2], 0-coherent varieties are shown to be exactly semi-abelian when viewed as categories (where they are characterised as pointed protomodular varieties). All 0-normal varieties built from groups (such as rings) are 0-coherent. So is the variety of Heyting algebras, and indeed of Heyting semilattices (Brouwerian semilattices) as was shown in $[9]$.

Let $\mathcal{A}$ be a 0 -normal variety. Obviously, if $A \in \mathcal{A}$ is 0 -coherent, it is join invariant as well: assume $M \triangleleft A$ in (1) of Proposition 4.1. Indeed, this also follows from the Mal'cev condition for 0-coherence given in [14], namely that a 0 -normal variety is 0 -coherent if and only if there is a term $\delta\left(x_{1}, x_{2}, \ldots, x_{n}, y_{1}, y_{2}, \ldots, y_{m}\right)$ and terms $p_{i}(x, y), i=1,2, \ldots, n$ such that for all such $i$, we have $p_{i}(x, x)=0$, and

$$
x=\delta\left(p_{1}(x, y), p_{2}(x, y), \ldots, p_{n}(x, y), y\right) .
$$

The Mal'cev condition in Theorem 2.2 yields the above, on letting the only $q_{j}$-term be $q_{1}(x, y)=y$.

Corollary 4.2. Every 0-coherent 0-normal variety is join invariant.

The converse of Corollary 4.2 fails. In the proof of Proposition 1.2, we showed that in the 0 -normal variety of EQ-semilattices, there are normals $I, J$ of an algebra such that $I \cdot J \neq J \cdot I$, so this variety is not even ideal determined. But the 0-normal variety of distributive EQ-lattices is much better behaved.

Proposition 4.3. The 0-normal variety of distributive EQ-lattices is join invariant but not 0-coherent.

Proof. Suitable terms for the variety of distributive EQ-lattices witnessing join invariance, as in Theorem 2.2 , are $\delta\left(x_{1}, y_{1}\right)=x_{1} y_{1}$, where $p_{1}(x, y)=$ $x \vee(x \bowtie y)$, so that $p_{1}(x, x)=x \vee 1=1$ and $q_{1}(x, y)=x \vee(y \bowtie 1)$. Hence, $\left.q_{1}(x, 1)=x \vee(1 \bowtie 1)=x \vee 1=1\right)$ and

$$
\begin{aligned}
\delta\left(p_{1}(x, y), q_{1}(x, y)\right) & =(x \vee(x \bowtie y))(x \vee(y \bowtie 1)) \\
& =x \vee(x \bowtie y)(y \bowtie 1) \\
& =x \vee x(x \bowtie 1)(y \bowtie 1)=x .
\end{aligned}
$$

Consider the distributive lattice of all subsets of $X=\{a, b, c\}$ under intersection and union, equipped with the topology given by the open sets $\mathcal{O}=$ $\{\emptyset,\{c\},\{b, c\}, X\}$. It is easy to check that $A=\{\emptyset,\{b\},\{c\},\{a, b\},\{b, c\}, X\}$ is a subalgebra, itself having $S=\mathcal{O} \cup\{\{b\}\}$ as a subalgebra. (Evidently, both contain $\mathcal{O}$, and hence are closed under $\bowtie$; closure under union and intersection is clear.) Then $I=\{\{c\},\{b, c\}, X\}$ is an ideal of $A$, indeed it is the principal 
filter of $\{c\}$. Now in $A,(\{b\} \bowtie\{a, b\})=\{b, c\} \in I$, so $\{a, b\} \in S \cdot I$ yet $S \vee I=S$, and so $S \cdot I \neq S \vee I$. Thus, this variety is not 0-coherent.

In [2], as a special case of a more general result applying to protomodular varieties, a characterisation of 0 -coherence of 0 -normal varieties is given in terms of subalgebras and homomorphisms. Thus, it was shown that $\mathcal{A}$ is 0 coherent if and only if the following holds for every algebra $A \in \mathcal{A}$ : for all $B \leq A$ for which there is a homomorphism $f: A \rightarrow B$ such that $f(b)=b$ for all $b \in B, \operatorname{ker}(f) \vee B=A$. This is the pointed version of 3 in [2, Proposition 2.1] and was used to obtain the Mal'cev condition for 0-coherence (or in fact the somewhat more general property of protomodularity considered there).

In fact, we have the following quite general fact.

Lemma 4.4. Let $\mathcal{A}$ be a 0 -regular variety, with $A \in \mathcal{A}$ and $B \leq A$.

Suppose there is a homomorphism $f: A \rightarrow B$ such that $f(b)=b$ for all $b \in B$. Then letting $K=\operatorname{ker}(f) \triangleleft A$, we have $B \cdot K=A$ and $B \cap K=\{0\}$.

Conversely, if $K \triangleleft A$ with $B \cap K=0$ and $B \cdot K=A$, then there exists a homomorphism $f: A \rightarrow B$ such that $f(b)=b$ for all $b \in B$, and $\operatorname{ker}(f)=K$.

Proof. Under the conditions of the first part of the lemma, let $a \in A$. Then $b=f(a) \in B$, so $f(b)=b=f(a)$, and then $a \rho_{K} b \in B$; thus, by definition, $a \in B \cdot K$. So $A \subseteq B \cdot K \subseteq A$ and so $B \cdot K=A$. If $a \in B \cap K$, then $a=f(a)=0$ since $K=\operatorname{ker}(f)$.

Conversely, if the conditions given in the second part of the lemma statement hold, note that $\rho_{K} \cap(B \times B)$ is the diagonal since its 0-class is $B \cap K=\{0\}$. For each $a \in A$, define $f(a)$ to be the unique $b \in B$ for which $(a, b) \in \rho_{K}$. (This $b \in B$ is unique since if also $\left(a, b^{\prime}\right) \in \rho_{K}$ for $b^{\prime} \in B$, then $\left(b, b^{\prime}\right) \in \rho_{K} \cap(B \times B)$, and so $b=b^{\prime}$.) It follows that $f$ is a homomorphism (from the fact that $\rho_{K}$ is a congruence), that $f(b)=b$ for all $b \in B$, and that the kernel of $f$ is $K$.

It now follows that the pointed version of 3 in [2, Proposition 2.1] can be re-expressed as follows:

For all $B \leq A$ and $K \triangleleft A$, if $B \cap K=\{0\}$ and $B \cdot K=A$, then $B \vee K=A$.

In turn, this condition is easily seen to be equivalent to:

For all $B \leq A$ and $K \triangleleft A$, if $B \cap K=\{0\}$, then $B \vee K=B \cdot K$.

So for 0-normal varieties, 0 -coherence is guaranteed by knowing that $M \cdot I=$ $M \vee I$ for all subalgebras $M$ and ideals $I$ for which $M \cap I=\{0\}$. Thus, we obtain a further equivalent condition to add to those characterising 0-coherent varieties of 0 -normal algebras.

We can also apply Lemma 4.4 to the join invariant setting, in the case in which it is additionally assumed that $B \triangleleft A$. This gives rise to characterisations of join invariant varieties analogous to those just given for 0 -coherent varieties.

Proposition 4.5. Let $\mathcal{A}$ be a 0 -normal variety. The following are equivalent.

(1) $\mathcal{A}$ is join invariant; 
(2) for all $B \triangleleft A$ for which there is a homomorphism $f: A \rightarrow B$ such that $f(b)=b$ for all $b \in B$, then letting $K=\operatorname{ker}(f) \triangleleft A, K \vee B=A$;

(3) for all $B, K \triangleleft A$ for which $B \cap K=\{0\}, B \cdot K=B \vee K$ (equivalently, $B+K=B \vee K)$.

Proof. (1) $\Leftrightarrow$ (2): follows from Lemma 4.4. (If $B \cdot K=B \vee K$, then $B \cdot K=K \cdot B$ and so $B \cdot K=B+K$.)

$(1) \Rightarrow(3)$ : This is immediate.

$(3) \Rightarrow(1)$ : This follows by noting that in the first direction of the proof of Theorem 2.2, the ideals $I_{x, y}$ and $I_{y, 0}$ have trivial intersection, so (3) can be deployed rather than the more general form in order to infer the existence of the Mal'cev terms given there.

So, analogous to the 0 -coherent case, at the variety level, it is sufficient to consider only ideals $I, J$ intersecting trivially when insisting that $I+J=I \vee J$ in order to ensure general join invariance.

\section{Join invariance and congruence permutability}

A fundamental property that a given variety of algebras may have is congruence permutability, also known as the Mal'cev property. There is a familiar Mal'cev condition for this property in terms of the existence of a ternary term.

A Mal'cev 0-regular variety $\mathcal{A}$ is ideal determined since certainly all congruences on every $A \in \mathcal{A}$ permute at zero. Conversely, not all ideal determined varieties are Mal'cev; for example, the variety of implication algebras is not (the example given in [6] of a non-Mal'cev ideal determined variety). At the other end of the spectrum, 0-coherent varieties are always Mal'cev as noted in [14], although not all 0-normal varieties with permutable congruences are 0 -coherent as shown in [8]. So the Mal'cev property lies strictly between the ideal determined and 0-coherence properties, sharing this feature with join invariance.

In this section, we explore the relationship between the Mal'cev and join invariance properties.

Consider the variety $\mathcal{V}$ of EQ-structures that are EQ-monoids equipped with one additional regular binary operation $t(x, y)$ satisfying the following laws:

- $t(x, 1)=1$;

- $t(x, x \bowtie y) t(x, y)=x$.

This variety is join invariant because we may set $p(x, y)=t(x, x \bowtie y), q(x, y)=$ $t(x, y)$, and $\delta(x, y)=x y$, as is easily seen.

In particular, in any distributive EQ-lattice $A$, define $t(x, y)=x \vee(y \bowtie 1)$ for all $x, y \in A$, and the above two laws are easily seen to hold. Also, for all 
$\alpha \in L_{A}$

$$
\begin{aligned}
\alpha \cdot t(x, y) & =\alpha(\alpha x \vee \alpha(y \bowtie 1))=\alpha(\alpha x \vee \alpha(\alpha y \bowtie 1)) \\
& =\alpha(\alpha x \vee(\alpha y \bowtie 1))=\alpha t(\alpha x, \alpha y),
\end{aligned}
$$

so $t(x, y)$ is a regular (binary) operation on $A$. Thus, the EQ-semilattice reduct of $A$ equipped with $t$ is in $\mathcal{V}$, as is any subalgebra of $A$ under the EQ-semilattice operations plus $t$.

Theorem 5.1. The 0-normal variety $\mathcal{V}$ has join invariance but is not Mal'cev.

Proof. Equip the set $X=\{a, b, c\}$ with the topology whose open sets are $\mathcal{O}=\{\emptyset,\{b\},\{a, b\},\{b, c\}, X\}$, and let $S=\mathcal{O} \cup\{\{a\},\{c\}\}$. Then $S$ is closed under the EQ-semilattice operations and $t(x, y)$ (which amounts to saying it is closed under the operation of taking the union of an arbitrary and an open subset, but not necessarily the union of two arbitrary subsets), and so $S \in \mathcal{V}$.

Consider the principal normal filters $I, J$ generated by the open subsets $\{a, b\}$ and $\{b, c\}$, respectively. Then $I=\{\{a, b\}, X\}, J=\{\{b, c\}, X\}$, and we see that the $\rho_{I}$-classes of $S$ are $I$ and $\{\emptyset,\{c\}\},\{\{a\}\},\{\{b\},\{b, c\}\}$, while the $\rho_{J}$-classes are $J$ and $\{\emptyset,\{a\}\},\{\{c\}\},\{\{b\},\{a, b\}\}$. So, $(\{c\},\{a\}) \in \rho_{I} \circ \rho_{J}$, but obviously $(\{c\},\{a\}) \notin \rho_{J} \circ \rho_{I}$. Thus, congruences in $S$ do not permute, and so $\mathcal{V}$ is not Mal'cev.

It is not known whether Mal'cev 0-normal varieties must have join invariance.

\section{Summary and Open Questions}

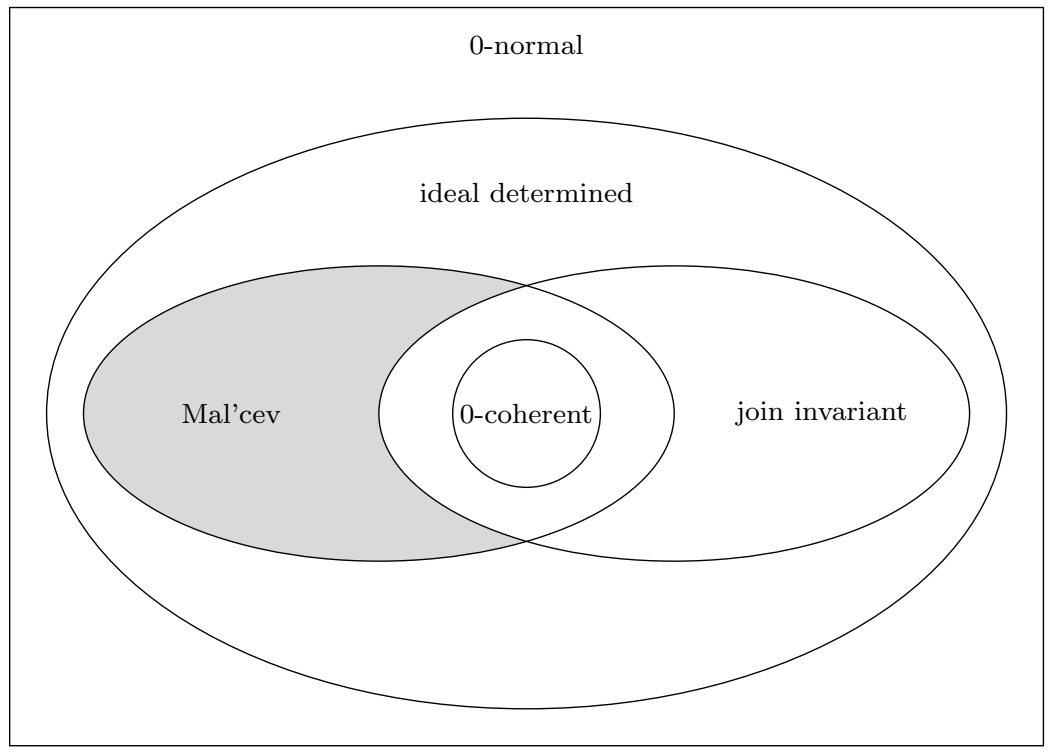


We have considered the following three properties of 0-normal varieties: (i) ideal determined, (ii) join invariant and (iii) 0-coherent, and shown that each defines a class that properly contains the next. We have also shown that there are join invariant varieties that are not Mal'cev, although we do not know whether Mal'cev 0-normal varieties must be join invariant.

It may be possible to somehow generalise the results of Section 3 to subtractive varieties satisfying the Mal'cev condition given in Theorem 2.2. Ideals do not correspond directly to congruences in general subtractive varieties, but the lattice of 0 -classes is a sublattice of the lattice of ideals.

Categorical formulations corresponding to ideal determined and 0-coherent varieties exist (ideal determined and semi-abelian categories, respectivelysee [8] and [7]). So there is interest in finding a categorical formulation of join invariance that reduces to join invariance when applied to varieties of algebras.

\section{REFERENCES}

[1] Beutler, E.: An ideal theoretic characterization of varieties of abelian groups. Algebra Universalis 8, 91-100 (1978)

[2] Bourn, D., Janelidze, G.: Characterization of protomodular varieties of universal algebras. Theory Appl. Categ. 11, 143-147 (2003)

[3] Burris, S., Sankappanavar, H.P.: A Course in Universal Algebra. http://www .math. uwaterloo.ca/ snburris/htdocs/ualg.html

[4] Fearnley-Sander, D., Stokes, T.: Varieties of equality structures. Internat. J. Algebra Comput. 13, 463-480 (2003)

[5] Fichtner, K.: Fine Bermerkung über Mannigfaltigkeithen universeller Algebren mit Idealen. Monatsh. d. Deutsch. Akad. d. Wiss. (Berlin) 12, 21-25 (1970)

[6] Gumm, H.P., Ursini, A: Ideals in universal algebras, Algebra Universalis 19, 45-54 (1984)

[7] Janelidzea, G., Márki, L., Tholen, W.: Semi-abelian categories. J. Pure Appl. Algebra 168, 367-386 (2002)

[8] Janelidzea, G., Márki, L., Tholen, W., Ursini, A.: Ideal-determined categories. Cahiers Topologie Géom. Différentielle Catég. LI-2, 115-125 (2010)

[9] Johnstone, P.: A note on the semiabelian variety of Heyting semilattices. In: Galois Theory, Hopf Algebras, and Semiabelian Categories. Fields Inst. Commun., vol. 43, pp. 317-318. Amer. Math. Soc. (2004)

[10] Köhler, P.: Brouwerian semilattices: the lattice of total subalgebras. Banach Center Publ. 9, 47-56 (1982)

[11] McConnell, N.R., Stokes, T.E.: Radicals of 0-regular algebras. Acta Math. Hungar. 113, 19-37 (2006)

[12] Stokes, T.E.: On EQ-monoids. Acta Sci. Math. (Szeged) 72, 471-496 (2006)

[13] Ursini, A.: Osservazioni sulle varieta BIT. Boll. Unione Mat. Ital. Sez. B Artic. Ric. Mat. (8) 7, 205-211 (1983) (Italian)

[14] Ursini, A., On subtractive varieties I. Algebra Universalis 31, 204-222 (1994)

\section{Nicholas McConnelL}

Department of Defence, Locked Bag 5076, Kingston, ACT 2604, Australia.

e-mail: nicholas.mcconnell@defence.gov.au

\section{Tim STOKES}

Department of Mathematics, The University of Waikato, Hamilton, New Zealand.

e-mail: stokes@waikato.ac.nz

$U R L:$ http://www.cs.uwinnebago.edu/homepages/menuhin 